\title{
Fault Detection for LPV Systems using Set-Valued Observers: A Coprime Factorization Approach
}

\author{
Daniel Silvestre $^{\mathrm{a}, *}$, Paulo Rosa ${ }^{1}$, João P. Hespanha ${ }^{1}$, Carlos Silvestre ${ }^{1}$ \\ ${ }^{a}$ Contact the author D. Silvestre through dsilvestre@isr.ist.utl.pt for a free \\ unformatted copy of this article or go to \\ https://www.sciencedirect.com/science/article/pii/S0167691117301019 for the final \\ formatted version.
}

\begin{abstract}
This paper addresses the problem of fault detection for linear parameter-varying systems in the presence of measurement noise and exogenous disturbances using Set-Valued Observers (SVOs). The applicability of current methods is limited in the sense that, to increase accuracy, the detection requires a large number of past measurements and the boundedness of the set-valued estimates is only guaranteed for stable systems. In order to widen the class of systems to be modeled and also to reduce the associated computational cost, the aforementioned issues must be addressed. A solution involving left-coprime factorization and deadbeat observers is proposed that reduces the required number of past measurements without compromising accuracy and allowing the design of SVOs for fault detection of unstable systems by using the resulting coprime factorization stable subsystems. The algorithm is shown to produce bounded set-valued estimates and an example is provided. Performance is assessed through simulations, illustrating, in particular that small-magnitude faults (compared to exogenous disturbances) can be detected under mild assumptions.
\end{abstract}

\section{Introduction}

The problem of detecting faults in the context of Linear Parameter-Varying (LPV) systems relates to that of determining if the current observations of the true system are compatible with the theoretical fault-free model. In particular, the framework of LPV systems is considered in this paper since applications of fault detection mechanisms for LPV systems are commonly found in industrial processes (see examples in the survey in [1]). In addition, distributed algorithms can also be viewed as LPV systems driven by dynamics dependent on stochastic or deterministic actions that can be measured only at the current time instant $[2]$.

\footnotetext{
* Corresponding author

Email address: dsilvestre@isr.ist.utl.pt (Daniel Silvestre)
} 
The study of fault detection problems has been a long standing research topic, since the early 70's (see [3]), but still poses remarkable challenges to both the scientific community and the industry (see, for example, the survey in [4] and the references therein). Classical fault detection methods such as the ones proposed in [3], [5], [6], [7], [8], [9] and [10], rely on designing filters that generate residuals that should be large under faulty conditions. These strategies aim to derive bounds (or thresholds) on these residuals that can be used to decide whether a fault has occurred or not. However, the computation of these thresholds is typically cumbersome or poses stringent assumptions on the exogenous disturbances and measurement noise acting upon the system. Many implementations of residual-based fault detection techniques are available in the literature such as [11], [12], [13] and [14].

In [15], [16], the authors develop the idea of using Set-Valued Observers (SVOs), whose concept was introduced in [17] and [18] (further information can be found in [19] and [20] and the references therein), for fault detection by resorting to a model invalidation (falsification) approach. The method is particularly interesting in the sense that it is able to handle a relatively large class of dynamic models, while also reducing the conservatism of the results by incorporating the information of past observations in the construction of the current set-valued state estimates. However, two main drawbacks of the approach can be identified: the convergence properties have only been proven for stable systems [16], and the calculation of the set-valued state estimates requires a significant computational effort. The latter limitation is a consequence of the need to increase the horizon of the observations to produce accurate results. The aim of this paper is to extend the SVO-based fault detection method in order to cope with unstable systems and to reduce the necessary horizon value for the class of LPV systems. This paper builds on existing results that exploit a left-coprime factorization-based approach into the design of SVOs for Linear Time-Invariant systems (LTI) [21].

The choice for representing the set of possible states depends on a mathematical formulation that enables fast and non-conservative intersections and unions of sets, as those are time-consuming operations when implemented in a computer. One alternative is to use the concept of zonotopes, described in [22] and further developed in [23],[24] and [25]. However, it is normally the case that each proposal represents a compromise between the computational complexity of the unions and intersections. Alternatively, the idea of interval analysis [26] may also be adopted, although it introduces conservatism by not considering horizon values larger than one in their typical formulation, unlike SVOs [16]. In [27], interval observers for linear and nonlinear systems are proposed with mild assumptions such as the boundedness of the disturbances and measurement noise (similar to the assumptions in this paper).

The main contributions of this paper can be summarized as follows:

- The use of a left coprime factorization for LPV systems enables SVO-based fault detection, even when the plant is unstable;

- The convergence proof of the method is provided for a broad class of LPV 
systems and for any horizon greater than $n_{x}$, the size of the state space, by exploiting the properties of deadbeat observers.

[1] W. J. Rugh, J. S. Shamma, Research on gain scheduling, Automatica 36 (10) (2000) $1401-1425$.

[2] D. Silvestre, P. Rosa, J. ao P. Hespanha, C. Silvestre, Stochastic and deterministic fault detection for randomized gossip algorithms, Automatica 78 (2017) 46 - 60. doi:http://doi.org/10.1016/j.automatica.2016.12.011. URL http://www.sciencedirect.com/science/article/pii/ S0005109816305192

[3] A. S. Willsky, A survey of design methods for failure detection in dynamic systems, Automatica 12 (6) (1976) $601-611$.

[4] I. Hwang, S. Kim, Y. Kim, C. Seah, A survey of fault detection, isolation, and reconfiguration methods, Control Systems Technology, IEEE Transactions on 18 (3) (2010) $636-653$.

[5] J. D. Barrett, Diagnosis and fault-tolerant control, Technometrics 49 (4) (2007) 493-494.

[6] J. Bokor, G. Balas, Detection filter design for LPV systems - a geometric approach, Automatica 40 (2004) 511-518.

[7] G. Ducard, Fault-tolerant Flight Control and Guidance Systems: Practical Methods for Small Unmanned Aerial Vehicles, Advances in industrial control, Springer, 2009.

[8] A. Marcos, S. Ganguli, G. J. Balas, An application of $\mathrm{H} \infty$ fault detection and isolation to a transport aircraft, Control Engineering Practice 13 (1) (2005) $105-119$.

[9] X. Ding, P. M. Frank, Fault detection via factorization approach, Systems and Control Letters 14 (5) (1990) $431-436$.

[10] S. Narasimhan, P. Vachhani, R. Rengaswamy, New nonlinear residual feedback observer for fault diagnosis in nonlinear systems, Automatica 44 (2008) 2222-2229.

[11] H. Hammouri, M. Kinnaert, E. E. Yaagoubi, Fault detection and isolation for state affine systems, European Journal of Control 4 (1) (1998) 2 - 16.

[12] D. Sauter, Diagnosis and fault-tolerant control m. blanke, m. kinnaert, j. lunze and $\mathrm{m}$. staroswiecki, springer-verlag: Berlin, 2003, $571 \mathrm{pp}$, isbn 3540-01056-4, International Journal of Robust and Nonlinear Control 15 (3) (2005) 151-154.

[13] J. Chen, R. J. Patton, Robust model-based fault diagnosis for dynamic systems, Vol. 3, Springer Science \& Business Media, 2012. 
[14] G. Ducard, Actuator fault detection in uavs, in: K. P. Valavanis, G. J. Vachtsevanos (Eds.), Handbook of Unmanned Aerial Vehicles, Springer Netherlands, 2015, pp. 1071-1122.

[15] P. Rosa, C. Silvestre, J. Shamma, M. Athans, Fault detection and isolation of LTV systems using set-valued observers, 49th IEEE Conference on Decision and Control (2010, Atlanta, Georgia, USA.) 768-773.

[16] P. Rosa, C. Silvestre, Fault detection and isolation of LPV systems using set-valued observers: An application to a fixed-wing aircraft, Control Engineering Practice 21 (3) (2013) $242-252$.

[17] H. Witsenhausen, Sets of possible states of linear systems given perturbed observations, IEEE Transactions on Automatic Control 13 (5) (1968) 556 -558 .

[18] F. Schweppe, Recursive state estimation: Unknown but bounded errors and system inputs, IEEE Transactions on Automatic Control 13 (1) (1968) $22-28$.

[19] F. Schweppe., Uncertain Dynamic Systems, Prentice-Hall, 1973.

[20] M. Milanese, A. Vicino, Optimal estimation theory for dynamic systems with set membership uncertainty: An overview, Automatica 27 (6) (1991) $997-1009$.

[21] P. Rosa, C. Silvestre, M. Athans, Model falsification using set-valued observers for a class of discrete-time dynamic systems: a coprime factorization approach, International Journal of Robust and Nonlinear Control 24 (17) (2014) 2928-2942.

[22] D. Bertsekas, I. Rhodes, Recursive state estimation for a set-membership description of uncertainty, IEEE Transactions on Automatic Control 16 (2) (1971) $117-128$.

[23] C. Combastel, A state bounding observer for uncertain non-linear continuous-time systems based on zonotopes, in: 44th IEEE Conference on Decision and Control, 2005 and 2005 European Control Conference. CDC-ECC '05., 2005, pp. 7228 - 7234.

[24] T. Alamo, J. Bravo, E. Camacho, Guaranteed state estimation by zonotopes, Automatica 41 (6) (2005) 1035 - 1043.

[25] J. K. Scott, D. M. Raimondo, G. R. Marseglia, R. D. Braatz, Constrained zonotopes: A new tool for set-based estimation and fault detection, Automatica 69 (2016) $126-136$.

[26] R. E. Moore, Interval analysis, Prentice-Hall series in automatic computation, Prentice-Hall, Englewood Cliffs, NJ, 1966. 
[27] T. Raissi, D. Efimov, A. Zolghadri, Interval state estimation for a class of nonlinear systems, Automatic Control, IEEE Transactions on 57 (1) (2012) 260-265. 